\title{
Machine Learning Assistant for Aircraft Acceptance
}

\author{
Rodrigo López Parra \\ Airbus, Paseo de John Lennon S/N, Getafe, Madrid (Spain) \\ rodrigo.I.lopez@airbus.com
}

\begin{abstract}
:
The task of testing an aircraft to be considered "ready for delivery" is a task that implies a lot of work, involvement of pilots, flight engineers and it demands significant costs. Optimize the time of acceptance flights is critical to save a lot of time and money. Currently the flight test engineers are responsible for supervising and planning all the maneuvers that are required to verify that all systems work properly.
\end{abstract}

The objective is to create a machine learning system which will analyze all the actions carried out in the aircraft during acceptance flights, and it will learn when to perform them, taking into account the maximum information available. This machine learning system must be able to perform the task currently performed by the flight engineer and lead the pilots in the maneuvers to be performed, also checking if they have been performed correctly.

Moreover, controlling thousands of variables that exist in an aircraft is only suitable for an artificial intelligence entity, which might detect possible problems and warn them with enough time to avoid major problems. This system could become an intelligent assistant essential in any environment.

Key words: machine learning, artificial intelligent, big data, deep learning, predictions

\section{Introduction}

New technologies are evolving at a very fast speed, as well as the programming paradigms that are progressing to achieve increasingly complex and smart systems.

Tab. 1: Programming paradigms

\begin{tabular}{|c|c|}
\hline \multicolumn{2}{|c|}{$\begin{array}{c}\text { A programming language is a } \\
\text { problem-solving tool }\end{array}$} \\
\hline $\begin{array}{c}\text { Imperative } \\
\text { Style Program }\end{array}$ & $\begin{array}{c}\text { algorithms + } \\
\text { data }\end{array}$ \\
\hline $\begin{array}{c}\text { Functional } \\
\text { Style Program }\end{array}$ & $\begin{array}{c}\text { functions * } \\
\text { functions }\end{array}$ \\
\hline Logic programming & facts + rules \\
\hline $\begin{array}{c}\text { Object-oriented } \\
\text { Style }\end{array}$ & $\begin{array}{c}\text { objects + mes- } \\
\text { sages }\end{array}$ \\
\hline
\end{tabular}

These have been the programming paradigms more important in the last decades.
However, we now have huge amounts of data, of which, new tools are needed that allow us to analyze hundreds or thousands of different variables at once, obtaining new results and knowledge.

Just as in his day, the pioneer Alan Turing created a machine to be able to evaluate the mathematical calculations much more quickly than any team of human beings, today we need machines that autonomously analyze all the millions of data we have in different contexts, and are able to detect anomalies where we do not see them, and even predict and recommend actions as any human being would do.

This is where the new tool development environments come into play to create automatic learning machines.

Machine learning applications make use of patterns in the data to make predictions rather than needing to be explicitly programmed. 


\section{Actual System, ASSPA}

Based on the acceptance programme for A400M aircraft manufactured on the final assembly line in Seville, Spain. There are currently more than 100 aircraft delivered to customers, which have had to go through a series of tests and flights to check every single part and system of the aircraft.

There is an acceptance manual that collects all the information with the results obtained in each of the tests and manoeuvres performed on each aircraft. This is the main basis for the customer to accept the aircraft.

Currently, we work with an application that uses the latest programming technologies called ASSPA (Automatic aSSistant Production Aircraft), which allows us to monitor and collect the necessary data from each maneuver performed.
We can see in Figure 1 the standard profile of an acceptance flight for an A400M aircraft.

This profile covers each of the maneuvers that pilots must perform at each altitude (flight level $\mathrm{FL}$ ).

And in Figure 2 we can see a data collection screen for the specific maneuver of Trim at FL $3 X X(3 X X 00$ feet $)$

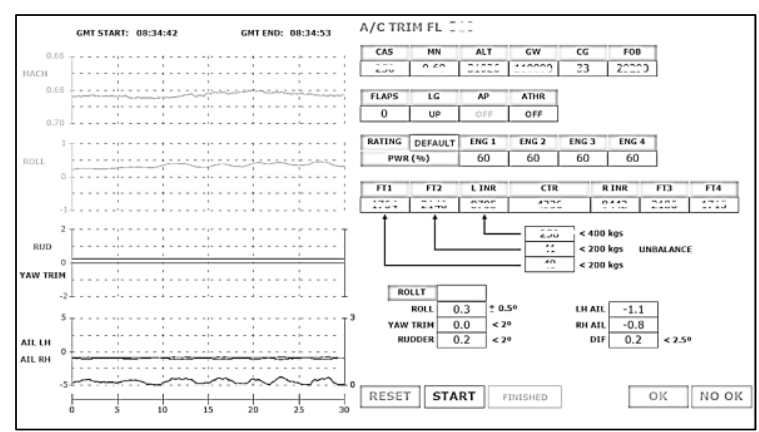

Fig 2. Check Point of AC Trim at FL3XX

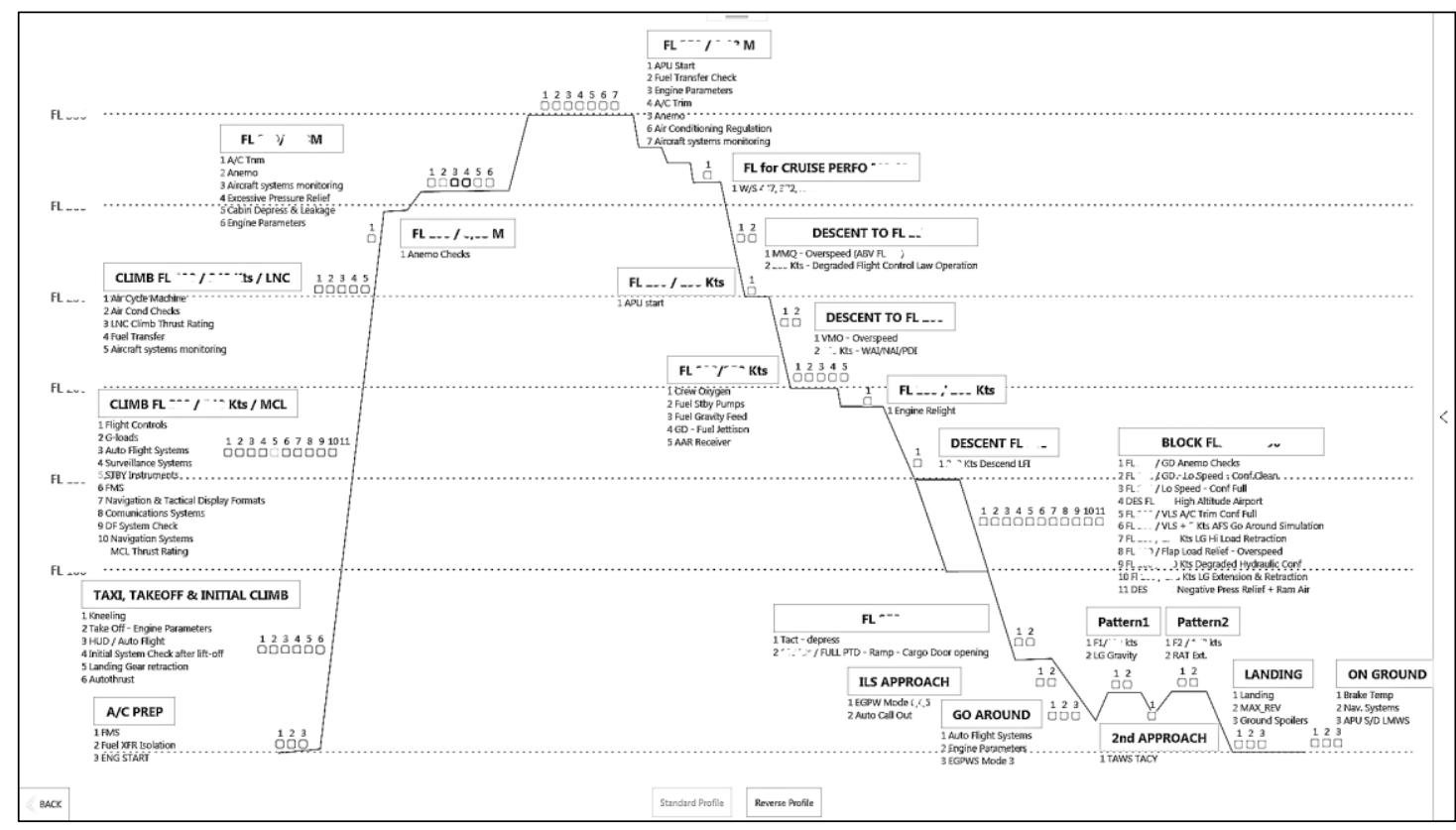

Fig 1. Standard Flight Profile for A400M Aircraft

This software helps flight engineers to collect data and manage the maneuvers that must be done at any moment. Collecting the data manually is practically impossible and each hour of flight is quite costly. In fact, this tool has become essential for aircraft acceptance tests. Great efforts have been made to automate the activation and data collection of certain manoeuvres without human intervention. For this purpose, certain variables such as flight phase, speed, altitude, engine power, data sequences, etc. have been taken into account with good results. Although taking into account all the casuistic that can occur in an aircraft, it is tremendously complex.

\section{Remark}

On the other hand, the aircraft's acquisition system is able to collect and record more than 250,000 different parameters during flights that can last up to 8 hours. Of these parameters, the ASSPA system uses only about 3000 parameters. That is, only $1.2 \%$ of the available data is used, and the current system can be considered quite advanced. A team of approximately 20-30 people is involved.

Who can extract the information we do not see from the $98.8 \%$ of data that is currently not used? 


\section{Machine Learning}

What is a Machine Learning?.

"A learning machine is one that learns by itself to find patterns without being specifically programmed by a human being."

This technology is at the heart of artificial intelligence and big data management.

Machine learning works by feeding an "algorithm or model" with input data that collects observations from the past, and builds a model to predict and classify new observations not known by the algorithm, imitating a human cognitive process.

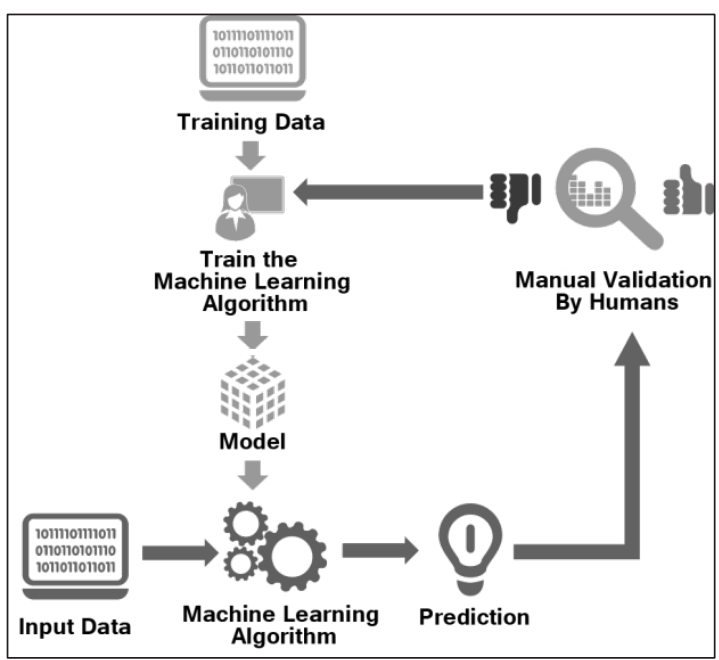

Ilustración 3. Machine Learning

Initially we do not know what we are looking for, we do not know what there is in that huge amount of data, we are not able to analyze parameter by parameter the 250,000 parameters and much less find the correlations between them if any. We need "something" to dive into all that data and look for "things" by itself that we don't have programmed.

A learning machine consists of three main parts:

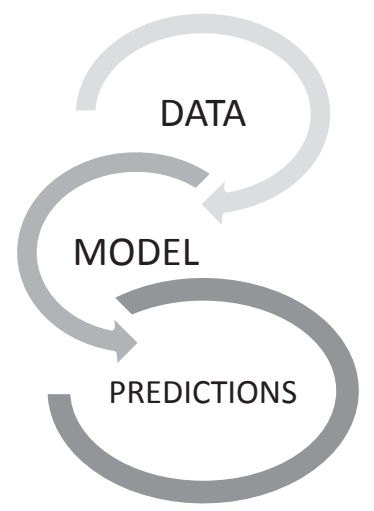

We have the data, although we could need to process it as input to the model.

The model is the "algorithms" that need to be trained with the data in order to make predictions.

And the predictions are the conclusions that the machine is able to detect by itself in the data.

There are four main types of learning algorithms in Machine Learning: supervised, unsupervised, reinforcement and deep learning.

Supervised learning is where you have input variables $(\mathrm{x})$ and an output variable $(\mathrm{Y})$ and you use an algorithm to learn the mapping function from the input to the output. $Y=f(X)$

The goal is to approximate the mapping function so well that when you have new input data (x) that you can predict the output variables $(Y)$ for that data.

For instance, regression and sales forecasting.

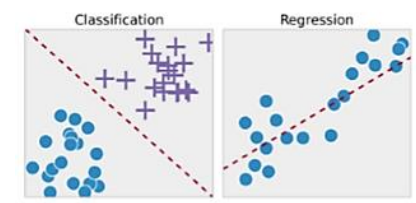

Ilustración 4. Classification, Regression

Unsupervised learning is where you only have input data (X) and no corresponding output variables.

The goal for unsupervised learning is to model the underlying structure or distribution in the data in order to learn more about the data.

For instance, Clustering, Splitting, ...

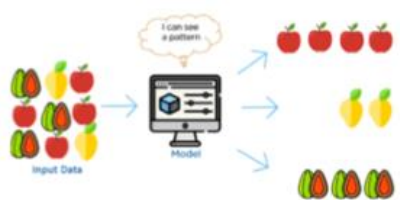

Ilustración 5. I can see a pattern

\section{Reinforcement}

Identify which actions a software agent should choose in a given environment in order to maximize some notion of "reward" or "penalty"

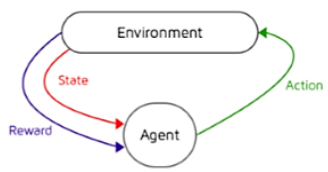

Ilustración 6. Reinforcement learning 
All these learning systems, allow us to classify, predict, form the way to act, and are very powerful tools. But we're looking for something even more advanced.

\section{Machine Learning - Deep Learning}

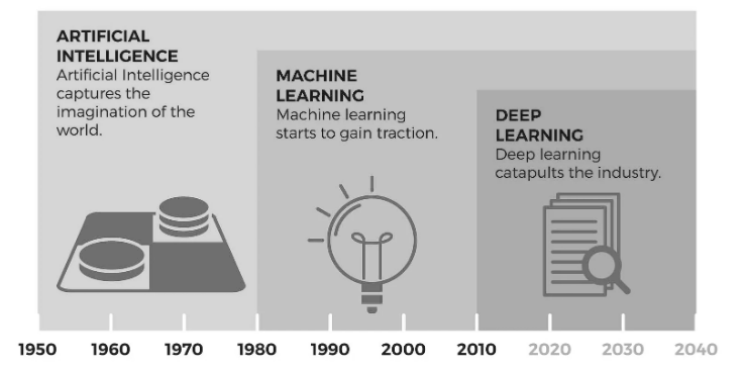

Ilustración 7. Evolution of artificial intelligence

In the figure we can see the evolution of $\mathrm{Al}$ (artificial intelligence) in the different decades.

While machine learning works with regression algorithms or decision trees, deep learning uses neural networks that function much like the biological neural connections in our brain.

It is extremely beneficial to data scientists too who are tasked with collecting, analyzing and interpreting large amounts of data.

The possibilities of creating learning machines for different purposes are endless. And it also depends on where our own imagination is able to reach. And it is in this field where we are interested.

\section{Anomaly detection}

We are at the beginning of our study to in a first step be able to detect anomalies in any equipment of the aircraft. Even be able to detect that an equipment is about to end its life time, before it fails completely.

\section{Example of $X-Y$ anomaly detection.}

In case of two-dimensional data ( $X$ and $Y$ ), it becomes quite easy to visually identify anomalies through data points located outside the typical distribution.

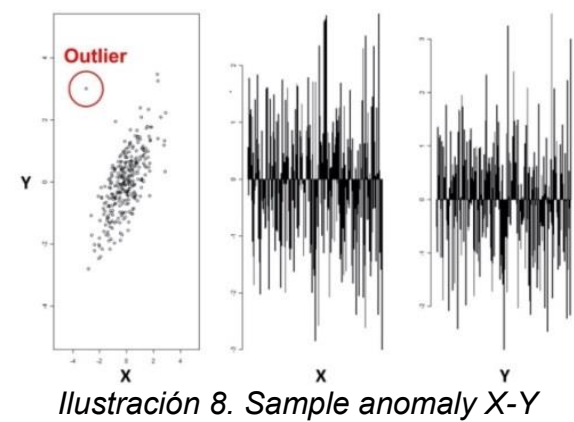

However, looking at the figures to the right, it is not possible to identify the outlier directly from investigating one variable at the time: It is the combination of the $X$ and $Y$ variable that allows us to easily identify the anomaly. This complicates the matter substantially when we scale up from two variables to $10-100$ s of variables, which is often the case in practical applications of anomaly detection and in our case.

Here is where we begin to see the need to create more complex learning machines, able to contrast and monitor hundreds or thousands of variables at once and find correlations between them.

\section{Neural Networks}

Essentially, neural networks are composed of layers of computational units called neurons, with connections in different layers. These networks transform data until they can classify it as an output. Each neuron multiplies an initial value by some weight, sums results with other values coming into the same neuron, adjusts the resulting number by the neuron's bias, and then normalizes the output with an activation function.

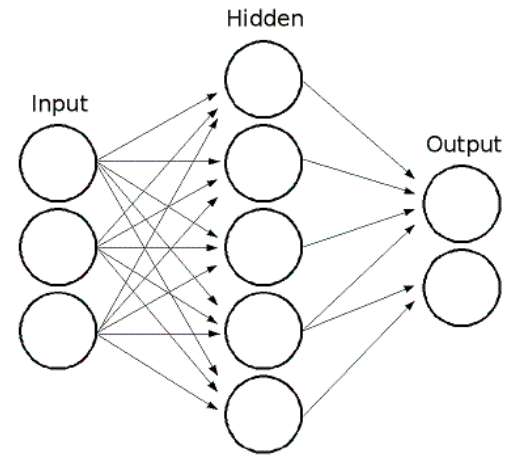

Ilustración 9. Neural Network

A key feature of neural networks is an iterative learning process in which records (rows) are presented to the network one at a time, and the weights associated with the input values are adjusted each time. After all, cases are presented, the process is often repeated. During this learning phase, the network trains by adjusting the weights to predict the correct class label of input samples. 


\section{Artificial Neural Network - Autoencoder}

This is a popular type of neural network with which we have started to create our first projects because could be fit with our goals.

An autoencoder is a type of artificial neural network used to learn efficient data codings in an unsupervised manner. The aim of an autoencoder is to learn a representation (encoding) for a set of data, typically for dimensionality reduction. Along with the reduction side, a reconstructing side is learnt, where the autoencoder tries to generate from the reduced encoding a representation as close as possible to its original input.

Architecturally, the simplest form of an autoencoder is a feedforward, non-recurrent neural network very similar to the many single layer perceptrons which makes a multilayer perceptron (MLP) - having an input layer, an output layer and one or more hidden layers connecting them - but with the output layer having the same number of nodes as the input layer, and with the purpose of reconstructing its own inputs.

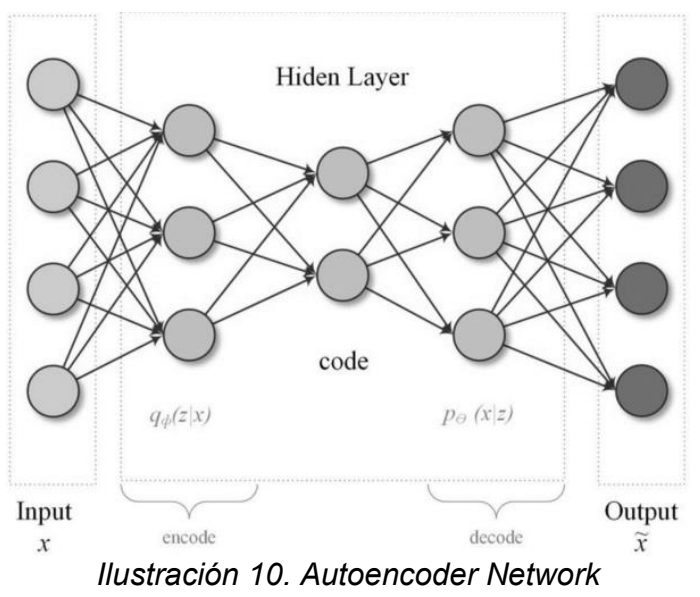

In the context of anomaly detection and condition monitoring, the basic idea is to use the autoencoder network to "compress" the sensor readings to a lower-dimensional representation, which captures the correlations and interactions between the various variables.

The autoencoder network is then trained on data representing the "normal" operating state, with the goal of first compressing and then reconstructing the input variables. During the dimensionality reduction, the network learns the interactions between the various variables and should be able to re-construct them back to the original variables at the output.

The main idea is that as the monitored equipment degrades, this should affect the interaction between the variables (e.g. changes in temperatures, pressures, vibrations, etc.). As this happens, one will start to see an increased error in the networks re-construction of the input variables. By monitoring the re-construction error, one can thus get an indication of the "health" of the monitored equipment, as this error will increase as the equipment degrades.

\section{Machine Learning for Aircraft Acceptance}

For our specific study we have data from new aircrafts, which we can choose those that we know have a perfect behavior of all systems, and that will be used to "train" our machine learning, to make it learn how is the correct behavior of each system.

Once our machine learning has been "trained" with this data, it can be used to check the correct functioning of the new aircraft that leave the factory, and to check the correct functioning during the whole life cycle of the aircraft.

We would have the perfect real-time guardian of the integrity of our entire aircraft.

And once this has been achieved, we will move on to a systems that are able of telling pilots when and how to act, without human intervention. The Machine Learning Assistant. 


\section{References}

[1] Vegard Flovik, Article, How to use machine learning for anomaly detection and condition monitoring. https://towardsdatascience.com/ (2018)

[2] Jay Shah, Article, Neural network learning. https://blog.statsbot.co (2017) 